\title{
ACERCA DE JASONIA HESPERIA MAIRE \& WILCZEK (ASTERACEAE) Y LAS ESPECIES NORTEAFRICANAS DE ESTE GÉNERO
}

\author{
Francisco GÓMIZ GARCÍA y Ramón MORALES VALVERDE
}

\begin{abstract}
RESUMEN. Acerca de Jasonia hesperia Maire \& Wilczek (Asteraceae) y las especies norteafricanas de este género. Se estudian las especies de Jasonia que viven en Marruecos, en especial J. hesperia. Se incluye una clave de identificación.
\end{abstract}

Palabras clave. Jasonia, Marruecos, Norte de África.

ABSTRACT. Jasonia hesperia Maire \& Wilczek (Asteraceae) and the species of the genus in North Africa. The species of the genus Jasonia living in Morocco are studied, especially J. hesperia. A identification key is included.

Key words. Jasonia, Morocco, North Africa.

\section{INTRODUCCIÓN}

El género Jasonia consta de siete especies que viven en el oeste de la región Mediterránea. Como ocurre con otros muchos géneros, falta un estudio preciso de las especies norteafricanas. Además este género tiene el inconveniente añadido de que sus especies florecen en verano u otoño, por lo cual se dispone aún de menos material del que sería de desear, dado que en dichas épocas del año suelen ser menos frecuentes las campañas de recolección.

Maire \& Wilczek (Maire, 1936) describieron como especie nueva Jasonia hesperia, planta recolectada en el jbel Tachilla y además publicaron una fotografía del pliego de la misma. Resulta amena la lectura del breve texto que acompaña al protólogo, pues sus autores nos relatan la pequeña peripecia que vivieron, para conseguir aquenios que les permitieran describir la especie de forma completa. En efecto, al tratarse de una planta cuya floración se produce en torno al equinoccio de otoño, aquel 10 de abril de 1935 en que la descubrieron, no existían más capítulos que los del año anterior, completamente secos y con la dispersión terminada. Vieron entonces un único capítulo en flor -una floración anormal- que fueron incapaces de alcanzar, pues lo producía una planta que crecía en un escarpe inaccesible. Finalmente encontraron un capítulo del año precedente, en el que una tela de araña había preservado para ellos los deseados aquenios y algunas 
flores secas que les permitieron describir la especie.

Desde entonces para acá, y si hemos de atenernos a lo publicado en la literatura botánica, pocas veces ha debido ser herborizada dicha planta. Emberger \& Maire (1941) añaden la localidad de Kerdous a la original, y así permanece su área de distribución hasta nuestros días. Brullo (1979: 303) da por buena la especie -aunque transfiriéndola al género Chiliadenus-, pero sin hacer mención alguna al material visto y limitándose a citar las localidades dadas por Emberger \& Maire casi cuarenta años antes. Dobignard et al. (1992), que exploraron el Antiatlas entre 1988 y 1989, aportando interesantes novedades corológicas, no mencionan la planta. Fennane \& Ibn Tattou (1998: 40) continúan dando esas dos mismas localidades como las únicas conocidas, citan la especie como endemismo de Marruecos y la consideran en la categoría de muy rara. Finalmente, Pardo de Santayana \& Morales (2004), incluyen provisionalmente la especie en cuestión como sinónimo de Jasonia rupestris Pomel, al no disponer de material, y basándose en la afinidad que ambas plantas presentan, según los autores de la que nos ocupa.

Tampoco uno de nosotros, que durante más de cinco años ha herborizado, con mayor o menor intensidad, en dicha cordillera, había conseguido encontrarla hasta abril de 2004, tropezando con el mismo inconveniente que encontraron sus descubridores en 1935.

Recientemente, y merced a la gentileza del buen amigo Makhluf Larbi, que vive en las inmediaciones de donde la planta crece, hemos podido disponer de material completo que nos permite afirmar y confirmar la independencia específica de $J$. hesperia frente a $J$. rupestris. Además damos la que es, según nuestros datos, la tercera localidad conocida de la misma.

Las otras tres especies que viven en el norte de África son J. rupestris, J. antiatlantica y $J$. glutinosa.

Aunque Vogt (2002) considera las especies que viven en el norte de Marruecos (J. rupestris y J. glutinosa) como pertenecientes a Chiliadenus, ya se ha discutido y justificado anteriormente por qué se consideran del género Jasonia (Pardo de Santayana \& Morales, 2004).

\section{METODOLOGÍA}

A partir del material disponible se ha elaborado una clave de las especies norteafricanas. En la enumeración de dichas especies se indican los datos relativos a estas, como el protólogo y su basiónimo, sinónimos, indicación locotípica, caracterización ecológica, intérvalo de altitud en que viven y períodos de floración con los meses señalados por números romanos, distribución, localidades conocidas y observaciones relativas a nomenclatura y caracteres botánicos. Dentro de las localidades conocidas se incluyen pliegos de herbario de MA y del herbario personal del primer autor; además se añaden las referencias conocidas de otros autores. Las figuras muestran fotografías de material de las tres especies exclusivas norteafricanas.

\section{RESULTADOS}

Aunque no se incluye más que una pequeña diagnosis en cada especie, con la siguiente clave de identificación son fáciles de identificar las cuatro especies que viven en Marruecos.

\section{Clave}

1. Hojas de elíptico-oblongas a lanceoladas, con indumento denso, seríceo-plateado. 1. J. hesperia 


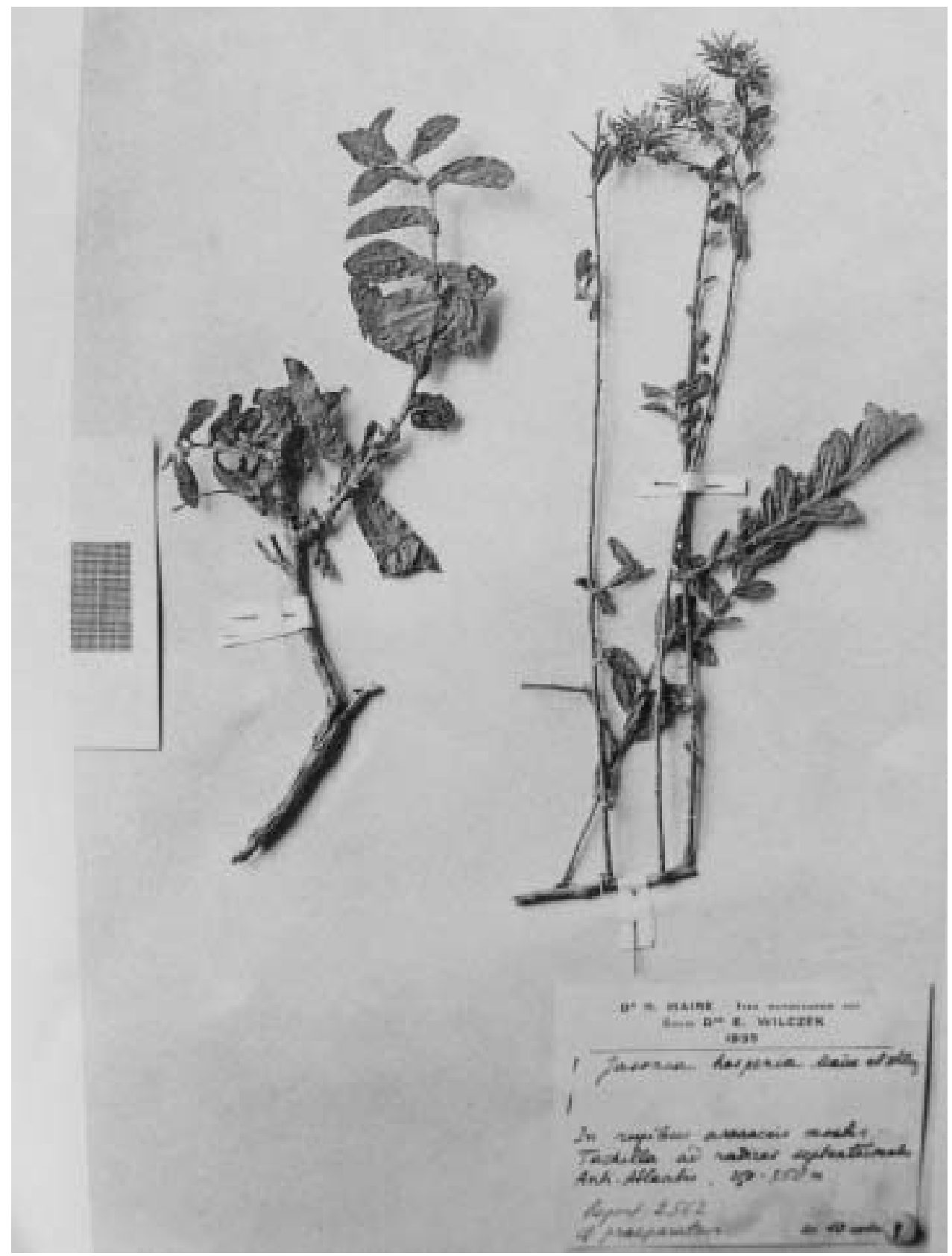

Figura 1. Pliego de Jasonia hesperia. Material tipo recolectado por Wilczek en 1935. [Transcripción de la etiqueta: $\mathrm{D}^{\mathrm{R}}$. R. Maire - Iter Maroccanum XXV / Socio D ${ }^{\mathrm{RE}}$ E. Wilczek / 1935 / Jasonia hesperia Maire et Wilez. / In rupibus arenaceis montis / Tachilla ad radices septentrionales / Anti - Atlantis, 250 - 350 m. / Repert. 2562 / d. praeparatum die 10 aprilis]. Sheet of Jasonia hesperia. Type material collected by Willckek in 1935. 
- Hojas de obovado-lanceoladas a linearlanceoladas, sin este indumento ................. 2

2. Tallos leñosos; hojas en general $<1 \mathrm{~cm}$; inflorescencia con ligulas rojizas

2. J. rupestris

- Tallos \pm herbáceos, frágiles; hojas en general > $1,5 \mathrm{~cm}$; inflorescencia con lígulas amarillas o solamente flosculada

3. Hojas caulinares linear-lanceoladas, de hasta $45 \times 5 \mathrm{~mm}$, longitud/anchura >6-7; inflorescencia con lígulas amarillas

3. J. antiatlantica

- Hojas caulinares lanceoladas de hasta 40 x 10 $\mathrm{mm}$, longitud/anchura <6-7; inflorescencia sin lígulas

4. J. glutinosa

\section{Relación de especies}

1. Jasonia hesperia Maire \& Wilczek in Maire, Bull. Soc. Hist. Nat. Afr. N. 27: 231-232 (1936)

Chiliadenus hesperius (Maire \& Wilczek) Brullo, Webbia 34: 303 (1979)

Ind. loc.: "Hab. in rupestribus arenaceis montis Tachilla ad radices boreo-occidentales Anti-Atlantis, ad. alt. 250-350 m, aestate et autumno florens."

Figs. 1, 2 y 3

Planta perenne, algo leñosa en la base. Hojas de elíptico-oblongas a lanceoladas, cubiertas de denso indumento seríceo-plateado. Capítulos con lígulas amarillas, en inflorescencia de inflorescencias congesta.

Grietas de rocas, 250-1250 m. IX. Endemismo de Marruecos que vive en el Antiatlas occidental.

\section{Localidades conocidas}

MARRUECOS: Anti-Atlantes: in rupestribus arenaceis montis Tachilla ad radices septentrionales, $250-350 \mathrm{~m}, 10-\mathrm{IV}-1935, E$. Wilczek 2562. Anti Atlas occidental (AA-1), jbel Tachilla (Fennane \& Ibn Tattou, 1998: 40; los autores se refieren a la cita anterior). Anti Atlas, Kerdous, Emberger (Emberger \& Maire, 1941: 1143). Anti Atlas occidental (AA-1), Kerdouss (Fennane \& Ibn Tattou, 1998: 40; los autores se refieren a la cita anterior). Antiatlas, en grietas de roquedos verticales del Jbel Imzi, $1250 \mathrm{~m}$, $9^{\circ} 17^{\prime} \mathrm{W} 29^{\circ} 45^{\prime} \mathrm{N}$ (29RMN7291), 6-IV-2004, F. Gómiz, Herb. F. Gómiz 6671. Antiatlas, en grietas de roquedos verticales del Jbel Imzi, $1250 \mathrm{~m}$,

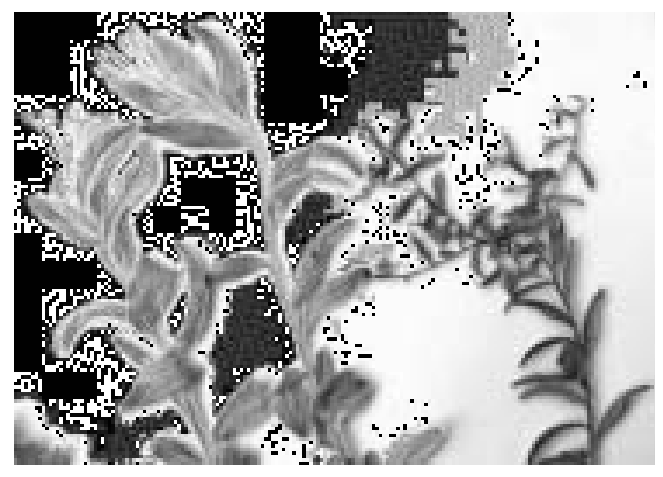

Figura 2.Hojas de J. hesperia. Leaves of J. hesperia.

$9^{\circ} 17^{\prime} \mathrm{W} 29^{\circ} 45^{\prime} \mathrm{N}$ (29RMN7291), 12-IX-2005, Makhluf Larbi, Herb. F. Gómiz 7359.

Observaciones. Es el número 2045 de la serie de Maire (1936: 231).

Fennane \& Ibn Tattou (1998: 40) incluyen este endemismo marroquí dentro de la categoría de especie muy rara. Solamente se conoce de las tres localidades indicadas arriba y comentadas en la introducción.

Además de las diferencias entre esta especie y $J$. rupestris, puestas ya de manifiesto por los autores que la describieron, lo primero que salta a la vista es el denso indumento seríceo-plateado que la cubre totalmente (figs. 2 y 3 ); carácter que no presenta $J$. rupestris (fig. 4). Lo más visible en segundo lugar, es que no es una planta tan sufruticosa y virgada, o erecta, como J. rupestris. Tampoco son parecidas sus inflorescencias, congestas en la primera y abiertas en la segunda. Algunos autores consideran como buen carácter diferencial el número de nervios del aquenio, 5 en $J$. hesperia y 10 en J. rupestris. Nosotros no hemos podido observar esta diferencia.

\section{J. rupestris Pomel, Nouv. Mat. F1. Atlantique} 1: 37 (1874)

Chiliadenus rupestris (Pomel) Brullo, Webbia 34: 302 (1979)

Ind. loc.: "Rochers calcaires et dolomitiques:

Tlemçen, Garrouban."

Fig. 4

Arbusto leñoso, pegajoso. Hojas obovado- 


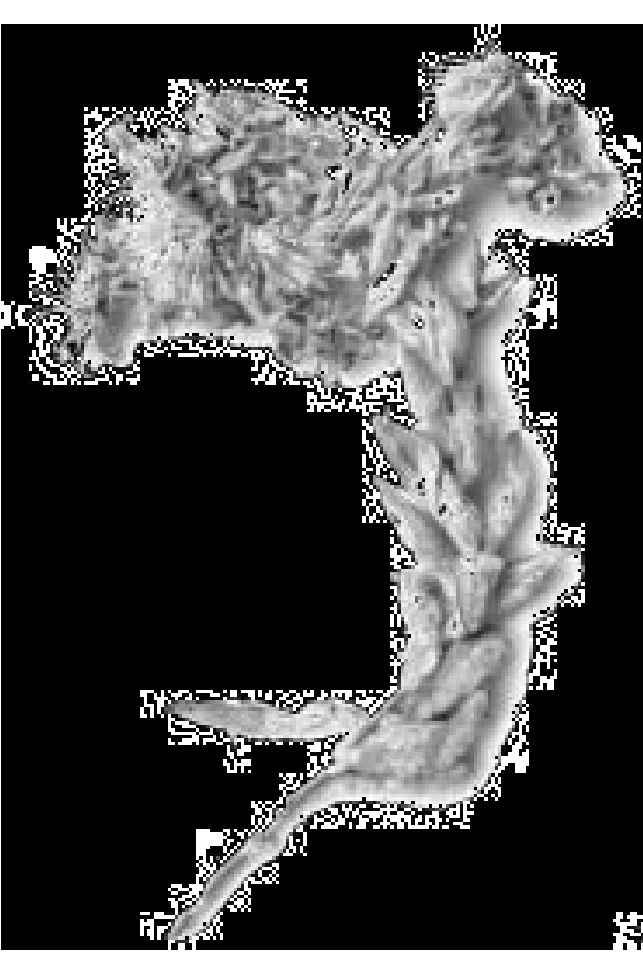

Figura 3. Detalle de parte superior e inflorescencia de $J$. hesperia. Detail of the upper part and inflorescence of J. hesperia.

lanceoladas, a veces muy pequeñas, $<1 \mathrm{~cm}$. Brácteas involucrales de tamaño heterogéneo. Capítulos con lígulas rojizas, en inflorescencia de inflorescencias más abierta.

Fisuras de rocas calizas, 150-240 m. IX-X. Región costera N de Marruecos y NW de Argelia.

\section{Localidades conocidas}

MARRUECOS: Imzourène, c. $50 \mathrm{~km} \mathrm{SE}$ of Al Hoceima, on road between Telat Azlat and Midar, $3^{\circ} 35^{\prime} \mathrm{W} 34^{\circ} 54^{\prime} \mathrm{N}$ (30S 446639386265 ), 240 m, limestone rocks, 27-X-1993, Jury 12840 \& Upson, MA 610947. Littoral de la Méditerranée (LM), monts de Kebdana (Fennane \& Ibn Tattou, 1998: 40). Littoral de la Méditerranée (LM), bords de la Moulouya et Melha (Fennane \& Ibn Tattou, 1998: 40). Littoral de la Méditerranée (LM), Al-Hoceima (Fennane \& Ibn Tattou, 1998: 40). Littoral de la Méditerranée (LM), Tidkirt (Fennane \& Ibn Tattou, 1998: 40). Rif Oriental, montes de Zaio, Quebdana y Ulad-Settut, in fissuris rupium, 24-IX-1920, C. Vicioso, MA 125877. Rif Oriental, Vado de Melha, UladSettut, in rupestribus, 23-IX-1920, C. Vicioso, MA 125878. Bocoia, Ras Sidi-el-Ahbed, 150 m, 15-X-1928, Font Quer Iter maroccanum 435, MA 125879. Taforalt, Gorges de Zezgel, 30SWD54, 600 m, 13-IX-1992, F. Gómiz, Herb. F. Gómiz 3527. Taforalt, Montes de Beni-Snassen, 30SWD55, 670 m, 13-IX-1992, F. Gómiz, Herb. F. Gómiz 3528. Monts du Maroc oriental, Bni Snassène (Om-1) (Fennane \& Ibn Tattou, 1998: 40).

Monts du Maroc oriental, Debdou (Om-3), monts de Debdou (Fennane \& Ibn Tattou, 1998: 40). Plaines et plateaux du Maroc oriental, basse Moulouya (Op-1), Oujda (Fennane \& Ibn Tattou, 1998: 40). Plaines et plateaux du Maroc oriental, basse Moulouya (Op-1), Taourirt (Fennane \& Ibn Tattou, 1998: 40). Plaines et plateaux du Maroc oriental, basse Moulouya (Op-1), montagnes entre Oujda et col de Jerada (Fennane \& Ibn Tattou, 1998: 40).

Observaciones.- Según Pomel (1874: 37) florece en VII-VIII y también se encuentra viviendo en dolomías. Este autor indica que las flores son de un color púrpura pálido.

Fennane \& Ibn Tattou (1998: 40) la consideran endemismo de Marruecos y Argelia, y la incluyen dentro de la categoría de especie rara.

3. J. antiatlantica (Emb. \& Maire in Maire) Gómiz, Anales Jard. Bot. Madrid 57(1): 191 (1999)

J. glutinosa var. antiatlantica Emb. \& Maire in Maire, Bull. Soc. Hist. Nat. Afr. N. 23: 189 (1932) [basión.]

Chiliadenus antiatlanticus (Emb. \& Maire) Gómiz, Anales Jard. Bot. Madrid 58(1): 199 (2000)

Ind. loc.: "Hab. in rupibus arenaceis nec non cristallinis Anti-Atlantis, ad. alt. 1400-1800 m: Agadir-n-Tigfert; Igherm (Emberger \& Maire, 1931)."

\section{Fig. 5}

Se caracteriza por sus raíces engrosadas, hojas linear-lanceoladas, de hasta 45 x $5 \mathrm{~mm}$, subpecioladas, con pelos sedosos y capítulos heterógamos, con flores femeninas periféricas liguladas (Gómiz, 1999: 191). 


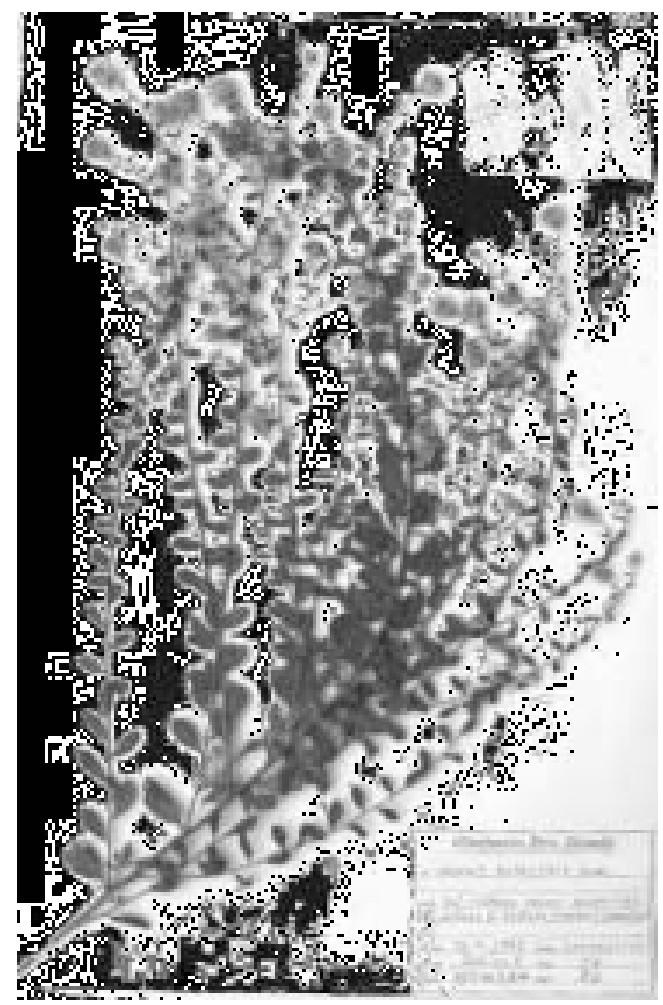

Figura 4. Pliego de J. rupestris. [Transcripción de la etiqueta: Herbario Fco. Gómiz / Jasonia rupestris Pomel / Hab. en terrerno rocoso dolomítico / Loc. Gorges de Zezgel (Taforalt) Marruecos / Fecha 13-9-1992 Fam. Compuestas / Cota 600 m Leg FG / UTM 30SWD54 Det FG]. Sheet of J. rupestris.

Roquedos silíceos; 1400-1800 m. VI-VIII. Endemismo de Marruecos, exclusivo del Antiatlas.

\section{Localidades conocidas}

MARRUECOS: Antiatlas, Jbel Kest, 29RMN8893, $1650 \mathrm{~m}$, terreno rocoso eruptivo silíceo, 25-VI-1999, F. Gómiz, MA 633139 (incluye diapositiva). Antiatlas, Jbel Kest, 29RMN8995, $1800 \mathrm{~m}$, terreno rocoso eruptivo silíceo, 26-VI1999, F. Gómiz, MA 633138. Anti-Atlas Kest (AA2), Agadir-n-Tigfert (Fennane \& Ibn Tattou, 1998: 40, como J. glutinosa). Anti-Atlas Kest (AA-2), Kest (Fennane \& Ibn Tattou, 1998: 40, como $J$.

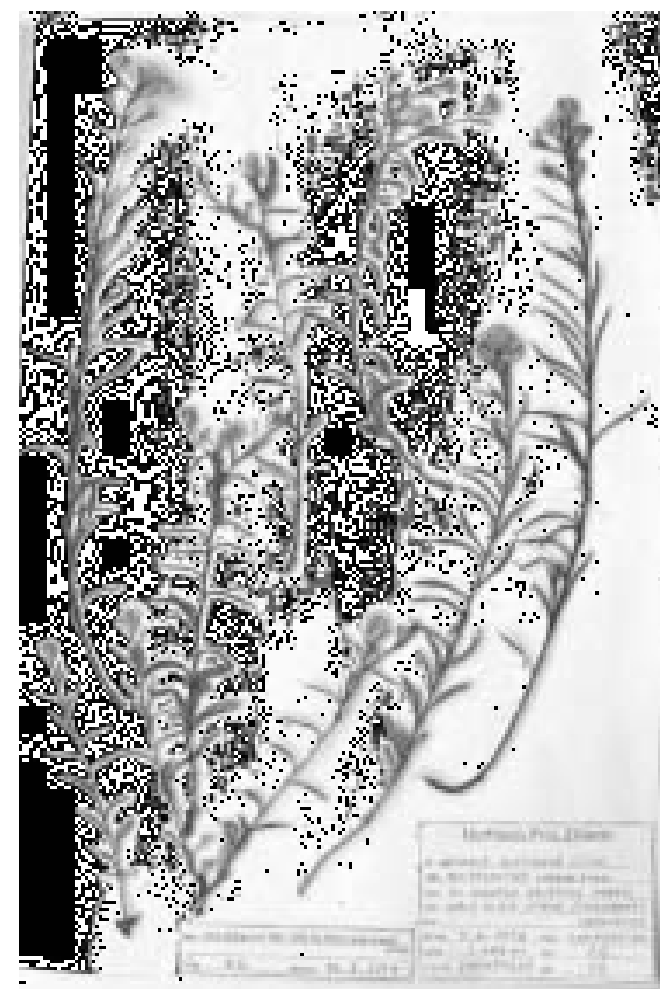

Figura 5. Pliego de J. antiatlantica. [Transcripción de la etiqueta: Herbario Fco. Gómiz / Sp. Jasonia glutinosa (L.) DC. / var. antiatlantica Emberger \& Maire / Hab. en roquedo eruptivo umbrío / Loc. Cerca de Ait Iftene (Taroudannt) / Nv. Maruecos / Fecha. 3-6-1998 Fam. Compuestas / Cota. 1.400 mts. Leg. F.G. / C.UTM. 29RMP9603 Det. F.G.]. Sheet of $J$. antiatlantica.

glutinosa). Anti-Atlas central (AA-3), Igherm (Fennane \& Ibn Tattou, 1998: 40; como $J$. glutinosa). Taroudannt, Ait Iftene, 29RMP9603, $1400 \mathrm{~m}$, en roquedo eruptivo umbrío, 3-VI-1998, F. Gómiz, Herb. F. Gómiz 5353.

Además se dispone de una fotografía en Gómiz (2001: 283, fot. 327), de jbel Kest, vertiente norte, Tizi-n-Tagounit.

Observaciones. Es el número 1254 de la serie de Maire (1932: 189).

4. J. glutinosa (L.) DC., Prodr. 5: 476 (1836) 
Erigeron glutinosum L., Mant. P1.: 112 (1767) [basión.]

Chiliadenus glutinosus (L.) Fourr., Ann.

Soc. Linn. Lyon, sér. 2, 16: 93 (1869)

Jasonia saxatilis (Lam.) Guss. Fl. Sicul. Syn. 2: 452 (1844)

Chiliadenus saxatilis (Lam.) Brullo, Webbia 34: 298 (1979)

Ind. loc.: "Habitat in Hispaniae, Galliae australis montosis maritimis"

Hojas caulinares lanceoladas, de hasta $40 \mathrm{x}$ $10 \mathrm{~mm}$. Capítulos sin lígulas, con flósculos amarillos.

Península Ibérica, S de Francia, Mallorca, Malta y N de Marruecos. VI-VIII (Vogt, 2002)

No se ha podido estudiar material de Marruecos, que es donde vive esta especie en el norte de África, aunque en España es especie bien conocida y utilizada como té (Pardo de Santayana $\&$ Morales (2004). Vogt (2002: 637) cita que vive en el oeste del Rif. Fennane \& Ibn Tattou (1998: 40) citan esta especie como rara. Las localidades que se indican a continuación son de dichos autores.

\section{Localidades conocidas}

MARRUECOS: Rif Tangérois (R-1), vallée de l'oued Laou (Fennane \& Ibn Tattou, 1998: 40). Rif centro-occidental (R-2), Bni-Selman (Fennane \& Ibn Tattou, 1998: 40). Rif centrooccidental (R-2), Bni-Zedjel (Fennane \& Ibn Tattou, 1998: 40). Rif centro-occidental (R-2), oued Madissouka (Fennane \& Ibn Tattou, 1998: 40). Rif centro-occidental (R-2), jbel Arhroud (Fennane \& Ibn Tattou, 1998: 40). Rif centrooccidental (R-2), jbel Bou Halla (Fennane \& Ibn Tattou, 1998).

AGRADECIMIENTOS. A Santos Cirujano, por la lectura crítica del manuscrito. A Antonio Martín Ciudad, por la ayuda técnica prestada.

\section{BIBLIOGRAFÍA}

BRULLO, S. -1979- Taxonomic and nomenclatural notes on the genera Jasonia Cass. and Chiliadenus Cass. -Compositae). Webbia 34(1): 289-308.
DOBIGNARD, A., F. JACQUEMOUD \& D. JORDAN -1992- Matériaux pour la connaissance floristique du Sahara occidental et de l'Anti-Atlas méridional. II. Leguminosae à Compositae. Candollea 47(2): 397-481.

EMBERGER, L. \& R. MAIRE -1941- Catalogue des plantes du Maroc (Spermatophytes et Ptéridophytes) 4. Supplément aux volumnes I, II et III. Alger.

FENNANE, M. \& M. IBN TATTOU -1998Catalogue des plantes vasculaires rares, menacées ou endémiques du Maroc. Bocconea 8: 5-243.

GÓMIZ, F. -1999- Notas sobre flora de Marruecos. Anales Jard. Bot. Madrid 57(1): 190-191.

GÓMIZ, F. -2000- Notas sobre flora de Marruecos. II. Anales Jard. Bot. Madrid 58(1): 199-200.

GÓMIZ, F. -2001- Flora selecta marroquí. Edición propia.

MAIRE, R. -1932-Contribution à l'étude de la Flore de l'Afrique du Nord. Fascicule 19. Bull. Soc. Hist. Nat. Afr. N. 23: 163-222.

MAIRE, R. -1936-Contribution à l'étude de la Flore de l'Afrique du Nord. Fascicule 24. Bull. Soc. Hist. Nat. Afr. N. 27: 203-238.

PARDO DE SANTAYANA, M. y R. MORALES 2004- Consideraciones sobre el género Jasonia (Compositae, Inuleae). Sistemática y usos. Acta Bot. Malacitana 29: 221-232.

POMEL, A. -1874- Noveaux Matériaux pour la Flore Atlantique 1. Paris, Alger.

VOGT, R. -2002- Chiliadenus in Valdés. Rejdali, Achmal El Kadmiri, Jury \& Montserrat (eds.). Catalogue des plantes vasculaires $d u$ Nord $d u$ Maroc, incluant des clés d'identification. CSIC. Madrid.

Aceptado para su publicación en mayo de 2006

Dirección de los autores. F. GÓMIZ: Apartado 1007.E-24080 León.e-mail: fgomiz@teleline.es. R. MORALES: Real Jardín Botánico. Plaza de Murillo, 2. 28014 Madrid. e-mail: morales@rjb.csic.es 